\title{
Assessment of Hybrid RANS/LES Turbulence Models for Aeroacoustics Applications
}

\author{
Veer N. Vatsa* \\ David P. Lockard ${ }^{\dagger}$ \\ NASA Langley Research Center, Hampton, VA 23681
}

\begin{abstract}
Predicting the noise from aircraft with exposed landing gear remains a challenging problem for the aeroacoustics community. Although computational fluid dynamics (CFD) has shown promise as a technique that could produce high-fidelity flow solutions, generating grids that can resolve the pertinent physics around complex configurations can be very challenging. Structured grids are often impractical for such configurations. Unstructured grids offer a path forward for simulating complex configurations. However, few unstructured grid codes have been thoroughly tested for unsteady flow problems in the manner needed for aeroacoustic prediction. A widely used unstructured grid code, FUN3D, is examined for resolving the near field in unsteady flow problems. Although the ultimate goal is to compute the flow around complex geometries such as the landing gear, simpler problems that include some of the relevant physics, and are easily amenable to the structured grid approaches are used for testing the unstructured grid approach. The test cases chosen for this study correspond to the experimental work on single and tandem cylinders conducted in the Basic Aerodynamic Research Tunnel (BART) and the Quiet Flow Facility (QFF) at NASA Langley Research Center. These configurations offer an excellent opportunity to assess the performance of hybrid RANS/LES turbulence models that transition from RANS in unresolved regions near solid bodies to LES in the outer flow field. Several of these models have been implemented and tested in both structured and unstructured grid codes to evaluate their dependence on the solver and mesh type. Comparison of FUN3D solutions with experimental data and numerical solutions from a structured grid flow solver are found to be encouraging.
\end{abstract}

\section{Introduction}

Despite significant progress made in the field of computational fluid dynamics (CFD) during the last several decades, prediction of noise sources around the landing gear of an aircraft is still an extremely challenging problem for the CFD community. The geometric complexity and highly irregular unsteady flow fields associated with landing gear pose numerous difficulties for numerical simulation. In order to better understand landing gear noise sources, it is instructive to examine simplified configurations that maintain some of the salient features of landing gear flow fields. It is for this reason that researchers in the past have examined the flow over single and multiple cylinder arrangements.

Unstructured grid methodology is gaining popularity in the CFD community with increasing emphasis on solving flow over more complex configurations. One of the objectives of this paper is to assess the performance and accuracy of the unstructured grid flow solver FUN3 $\mathrm{D}^{1}$ for aeroacoustics applications by comparisons with solutions obtained with a well-established structured grid flow solver, such as CFL3D, ${ }^{2}$ and experimental data for some benchmark problems.

A substantial database consisting of surface and off-body measurements has been generated for cylinders at the Basic Aerodynamic Research Tunnel (BART) ${ }^{3}$ and the Quiet Flow Facility (QFF) ${ }^{4}$ at NASA Langley Research Center. Unsteady Reynolds-averaged Navier-Stokes (RANS) solutions obtained with the block-structured grid flow solver CFL3D corresponding to several of the configurations examined in these experiments are available in Refs. 5 and 6. Based on such simulations, it became clear that the standard turbulence models developed for steady flows, such as the Spalart-Allmaras (SA) one equation turbulence $\operatorname{model}^{7}$ and Menter's two-equation shear stress transport (SST) turbulence model $^{8}$ are overly diffusive and do not produce the small-scale structures observed experimentally in wake regions of such configurations. Full 3-D simulations with some form of hybrid turbulence model, where the growth of eddy viscosity is controlled in reverse flow regions, are essential to capture the small-scale structures needed to accurately predict such flows. Khorrami et al. ${ }^{9}$ and Lockard et al. ${ }^{6}$ modified the SST turbulence model in the CFL3D code by turning off the production terms outside the boundary layer region to control the growth of turbulence and obtained good comparisons with the experimental data of Refs. 3 and 4.

In this paper, we examine some of the hybrid turbulence models that have become available recently. In general, we are seeking turbulence models that are amenable to grid refinement and are applicable to many different complex geometries without a need for adjusting input parameters from case to case. We are also interested in comparing the results

\footnotetext{
* Senior Research Scientist, Computational Aerosciences Branch, Research Directorate; Associate Fellow AIAA

${ }^{\dagger}$ Senior Research Scientist, Computational Aerosciences Branch, Research Directorate,; Senior Member AIAA

This material is declared a work of the U.S. Government and is not subject to copyright protection in the United States.
} 
from multiple CFD codes with different turbulence models. In particular, we are seeking to calibrate an unstructuredgrid code for cylinder configurations before applying this methodology to more complex geometries, such as realistic landing gear configurations.

\section{Experiments}

Hutcheson et al. ${ }^{4}$ and Jenkins et al. ${ }^{3,10}$ have performed experimental studies for single and tandem cylinder configurations in the QFF and BART tunnels at NASA Langley Research Center. These experimental studies produced a large database consisting of steady surface pressures, detailed off surface measurements of the flow field using Particle Image Velocimetry (PIV), hot-wire measurements in the downstream wake, and unsteady pressure data. Although the cylinder diameter varied between some of the tests, the free-stream conditions were set to achieve a Reynolds number based on cylinder diameter of $1.66 \times 10^{5}$. A transition strip was placed between azimuthal locations of 50 and 60 degrees from the front stagnation point to excite a fully turbulent shedding process. More complete details of the experimental set up are available in Refs. 3,4,6 and 10.

\section{Governing Equations and Flow Solvers}

In the present work, FUN3D and CFL3D, two widely used second-order accurate CFD codes, are used to obtain numerical solutions of the unsteady Reynolds-averaged Navier-Stokes (RANS) equations. Both of these codes have a variety of flux schemes and flux limiters to choose from. For the current work, Roe's flux-difference splitting scheme ${ }^{11}$ is used without a flux limiter.

FUN3D is an unstructured grid flow solver developed originally by Anderson and Bonhaus. ${ }^{12}$ The FUN3D code has gone through significant modifications over the years by a team of researchers at NASA Langley using modern software practices. ${ }^{1}$ The discrete form of the governing equations are solved either in a time-accurate manner with a constant time step at every grid point or with variable time stepping to accelerate convergence to a steady state. At each iteration step, the linear system of equations is relaxed in a red-black fashion with a point implicit procedure. ${ }^{13}$ The FUN3D code can accommodate multiple grid types including tetrahedrons, prisms, pyramids and hexahedrons, which makes it compatible with many different grid generators.

The multi-block, structured grid flow solver, CFL3D ${ }^{2}$ (version 6) developed at NASA Langley Research Center is used to solve the 3-D, time-dependent, RANS equations using a finite-volume formulation, where the viscous fluxes in all three grid coordinates are retained. The calculations use the third-order upwind biased scheme with flux-difference splitting, which has been shown to provide second-order spatial accuracy in previous applications of CFL3D.

Both of these codes employ a dual time-stepping algorithm with subiterations used to converge the solution within each time step. We normally used between 15-30 subiterations per time step, but the number varies for different grids. A variety of time marching schemes are available in FUN3D, including a second order backward differencing formulation (BDF2), and an optimized second order backward differencing formulation (BDF2OPT). The CFL3D code uses the $\mathrm{BDF} 2$ formulation for unsteady problems.

\section{Configurations and Grids}

The current numerical simulations of circular cylinder configurations are assumed to be in free air without any tunnel walls. Here, the $\mathrm{x}$-coordinate is aligned with the free-stream direction and the $\mathrm{z}$-coordinate is along the spanwise direction. For the single cylinder, the far-field boundary is set at a distance of 30 cylinder diameters, or $30 \mathrm{D}$ ( $\mathrm{D}$ being the cylinder diameter) away from the center of the circular domain. Based on the work of Lockard et al., ${ }^{6}$ a span of 3 cylinder diameters is used which is considered the minimum value for the spanwise extent to capture the three-dimensionality in such problems. A structured grid consisting of 289 x 289 points in the circumferential and radial directions was created in a two-dimensional plane. This grid was extruded to produce 97 equally spaced planes in the spanwise direction to generate the three-dimensional grid used in these computations. The grid was clustered near the cylinder surface with a normal grid spacing equal to $0.0002 \mathrm{D}$ which results in a value of 1-2 for the non-dimensional turbulent wall distance, $y^{+}$. A partial view of the planar grid is shown in Fig. 1(a), where the grid clustering near the surface and wake region is clearly visible. The choice of grid clustering in the wake region was influenced by the Detached Eddy Simulation (DES) arguments proposed by Spalart ${ }^{14}$ and to replicate the grid topology suggested for DES simulations for cylinders by Travin et al. ${ }^{15}$ and Vatsa and Singer. ${ }^{16}$

For the tandem cylinders, the configuration examined here is comprised of two cylinders of equal diameter aligned along the streamwise direction and separated by a distance of 3.7 D. For the CFL3D code, a structured grid of medium 
resolution described in an earlier paper by Lockard et al. ${ }^{6}$ was used. Because portions of this grid are not point-matched at block interfaces which create hanging nodes, this grid could not be used directly in the FUN3D unstructured grid flow code. An attempt was made to create a hybrid grid, where the point-matched portion of the CFL3D grid in the near-field was retained and the far-field region was filled with an unstructured grid. However, we encountered numerical difficulties in obtaining FUN3D solutions on this grid with two-equation based turbulence models. As an alternative, a two-dimensional hybrid grid consisting of a structured grid in the boundary layer regions surrounding the cylinders and then transitioning into unstructured grid was created using the AFLR $2^{17}$ grid generator. The grid spacing near the cylinder surfaces is approximately $0.0002 \mathrm{D}$, as was the case for the structured grid. The outer boundary was placed approximately $25 \mathrm{D}$ away from the center of the computational domain. An attempt was made to keep the grid density in the near wake regions and regions close to the cylinders comparable to the medium resolution structured grid used in Ref. 6. This sectional grid, shown in Fig. 1(b), was then extruded to 97 planes in the spanwise (z) direction to generate a three-dimensional grid for a span of three diameters $(3 \mathrm{D})$.

\section{Turbulence Models}

An attempt is made in this paper to examine the performance of several popular turbulence models for computing the unsteady flow over the cylinder configurations discussed earlier. The first model considered here is based on the shear stress transport (SST) model of Menter. ${ }^{8}$ For the current applications, the SST model is modified following the quasi-laminar approach introduced by Khorrami et al. ${ }^{9}$ and used more recently by Lockard et al. ${ }^{6}$ This model will be referred as the "SST-QL" model in this paper. Essentially, the production terms in the SST model are set to zero at a preselected distance away from the cylinder wall. In the CFL3D code, the switch is made at a grid index in the wall normal direction, such that the distance from the wall where production terms are zeroed out varies between 0.01D (near the front stagnation point) to $0.04 \mathrm{D}$ (near the top and bottom of cylinders). In the FUN3D code, the switch is tied directly to the distance normal to the wall. For the present calculations, the production terms are set to zero at a distance of $0.03 \mathrm{D}$ away from the wall, so that the near wall flow is governed by the standard RANS model for turbulence. Despite the successful use of the SST-QL model in Refs. 6 and 9, it is recognized that this formulation is strongly dependent on grid topology and difficult to generalize for complex configurations consisting of multiple components.

The next turbulence model under consideration is formed by a linear combination of the two-equation SST model ${ }^{8}$ developed for RANS equations and an LES subgrid scale model as described by Lynch and Smith. ${ }^{18}$ This turbulence model is available in the FUN3D code and will be referred to as hybrid RANS/LES or "HRLES" model. Such hybrid models are gaining broader acceptance in the CFD community because they incorporate the effect of unsteady flow physics. In addition, these hybrid models are relatively independent of grid topology and therefore should be applicable to a wider range of configurations.

Another turbulence model under consideration is based on the one-equation Detached Eddy Simulation (DES) model of Spalart. ${ }^{14}$ Although the DES model has been used often for solving unsteady separated flows, in our experience this model depends too strongly on the grid quality and topology, and can lead to non-physical results with grid refinement in viscous layers. Spalart et al. ${ }^{19}$ have recently modified the DES model to overcome some of the shortcomings related to its grid dependence in a new model named Delayed Detached Eddy Simulation or DDES. The DDES model applies a blending function based on $f_{d}$ (defined later in this section) to the destruction terms in the turbulence equation. The function $f_{d}$ varies between 0 for RANS mode and 1 for LES mode. We detected pockets of large eddy-viscosity in regions upstream of the cylinders in our initial application of the DDES model. In order to overcome such non-physical behavior, the blending function is also applied to the production terms. However, to retain the same near wall behavior, the function is only applied to the production terms when $f_{d}>C_{M D D}$. The new blending function has the form

$$
f_{m}=\frac{\left(1-f_{d}\right)}{1-C_{M D D}}
$$

The constant $C_{M D D}$ should be close to unity. We have incorporated this modified DDES model, named MDDES, in both the CFL3D and FUN3D codes, and the resulting solutions using $C_{M D D}=0.975$ are included in this paper. We have run other tests with $C_{M D D}=0.9975$ with some indication that the higher value is better, but more calibration studies are needed. The modified turbulence model can be written in dimensional form as

$$
\frac{\partial \tilde{\nu}}{\partial t}+u_{j} \frac{\partial \tilde{\nu}}{\partial x_{j}}=c_{b 1}\left(1.0-f_{t 2}\right)\left(\Omega f_{m}+\hat{s}\right) \tilde{\nu}-\left[c_{w 1} f_{w}-\frac{c_{b 1}}{\kappa^{2}} f_{t 2}\right]\left(\frac{\tilde{\nu}}{\tilde{d}}\right)^{2}+\frac{1}{\sigma}\left[\frac{\partial}{\partial x_{j}}\left((\nu+\tilde{\nu}) \frac{\partial \tilde{\nu}}{\partial x_{j}}\right)+c_{b 2} \frac{\partial \tilde{\nu}}{\partial x_{i}} \frac{\partial \tilde{\nu}}{\partial x_{i}}\right]
$$

and the turbulent eddy viscosity is computed from

$$
\mu_{t}=\rho \tilde{\nu} f_{v 1}
$$


where

$$
\begin{gathered}
\chi=\frac{\tilde{\nu}}{\nu}, \quad \tilde{S}=\Omega+\hat{s}, \hat{s}=\frac{\tilde{\nu}}{\kappa^{2} \tilde{d}^{2}} f_{v 2}, \quad f_{v 1}=\frac{\chi^{3}}{\chi^{3}+c_{v 1}^{3}}, \quad f_{v 2}=1-\frac{\chi}{1+\chi f_{v 1}}, f_{w}=g\left[\frac{1+c_{w 3}^{6}}{g^{6}+c_{w 3}^{6}}\right]^{1 / 6}, \\
g=r+c_{w 2}\left(r^{6}-r\right), \quad r=\min \left[\frac{\tilde{\nu}}{\tilde{S} \kappa^{2} \tilde{d}^{2}}, 10\right], \quad f_{t 2}=c_{t 3} \exp \left(-c_{t 4} \chi^{2}\right), \quad r_{d}=\frac{\nu_{t}+\nu}{\sqrt{U_{i, j} U_{i, j}} \kappa^{2} d^{2}}, \\
f_{d}=1-\tanh \left(\left[8 r_{d}\right]^{2}\right), \tilde{d}=d-f_{d} \max \left(0, d-C_{D E S} \Delta\right)
\end{gathered}
$$

Here, $\rho$ is the density, $\nu=\mu / \rho$ is the molecular kinematic viscosity, $\mu$ is the molecular dynamic viscosity, $\Omega$ is the magnitude of the vorticity, $U_{i, j}=\frac{\partial U_{i}}{\partial x_{j}}$ is the (i,j)'th component of stress tensor, and $d$ is the distance from the field point to the nearest wall. Additional definitions are given by:

$$
\begin{gathered}
\tilde{\nu}_{\text {wall }}=0, \quad \tilde{\nu}_{\text {farfield }}=3 \nu_{\infty}, \quad c_{b 1}=0.1355, \quad \sigma=2 / 3, \quad c_{b 2}=0.622, \quad \kappa=0.41, \\
c_{w 1}=\frac{c_{b 1}}{\kappa^{2}}+\frac{1+c_{b 2}}{\sigma}, \quad c_{w 2}=0.3, \quad c_{w 3}=2, \quad c_{v 1}=7.1, \quad c_{t 3}=1.2, \quad c_{t 4}=0.5, \quad C_{D E S}=0.65
\end{gathered}
$$

\section{Results}

The computations were performed at a free-stream Mach number of 0.166 and a Reynolds number of $1.66 \times 10^{5}$ based on the cylinder diameter to match the test conditions of the experimental study of Jenkins ${ }^{3}$ in the BART tunnel at NASA Langley. As mentioned earlier, a transition strip was used in the experiments to ensure turbulent separation and shedding around the cylinders. The computations were run in a fully turbulent mode, and in general, transition to turbulent flow occurred downstream of the trip locations but ahead of the observed separation. Hence, in the simulations, flow separation occurred in the turbulent regime.

Adiabatic wall conditions are imposed on the surface of the cylinder and Riemann-invariant based boundary conditions are imposed on the far-field boundary. In the spanwise direction, periodic boundary conditions are imposed at $z=0$ and $z=z_{\max }$ to more closely simulate an infinite span problem.

\section{VI.A. Single Cylinder}

The first set of results presented here are for turbulent flow past an isolated circular cylinder. Normally, the CFD code is initially run in a steady mode to expedite the development of oscillatory solutions. We then switch to a timeaccurate mode to capture the unsteady behavior of the flow field. The simulations are run over long periods of time for aeroacoustics applications and care must be taken to ascertain that the numerical solutions are converged well within each time step sub-iteration loop. In addition, the computational time step must be chosen carefully such that the temporal errors do not pollute the solution accuracy. The temporal accuracy for the single cylinder case was examined recently by Vatsa et al. ${ }^{20}$ for a family of backward difference (BDF) schemes. Based on this study it was concluded that the non-dimensional time step of $\Delta t c_{\infty} / D=0.102$ (corresponding to 260 time steps per period for the primary shedding frequency) chosen here produces accurate solutions with the BDF2OPT scheme used in the FUN3D solver. Here, $c$ is the speed of sound.

A snapshot of the residual convergence for the mass conservation and turbulence kinetic energy equations in the dual time-stepping scheme from a FUN3D computation is shown in Fig. 2(a). Approximately 4 orders of residual reduction are seen for the mass conservation equation, which is considered adequate for keeping temporal errors from degrading the solutions. The turbulence kinetic energy (TKE) equation also displays good convergence and low residuals in this figure. Similar convergence levels are observed in the CFL3D solutions.

The variation of the lift and drag coefficients for the cylinder with time are presented in Fig. 2(b). This figure clearly shows the aperiodic nature of force coefficients and the need for large number of time iterations in order to achieve statistically meaningful solutions. As expected, the lift coefficient oscillates around a mean value of zero, and the drag coefficient oscillates about a positive number.

Next we examine the time-averaged pressure distributions on the cylinder surface. We started averaging the flow field data after running the code for 20,000 time steps $(0.26$ secs.) to wash the initial disturbances out of the computational domain. The solutions were run for at least another 60,000 time steps to achieve statistically meaningful results. The time-averaged surface $\mathrm{Cp}$ distribution at each circumferential location was also averaged in the spanwise direction to extract the time-averaged data as a function of the circumferential angle $\theta$. The angle $\theta$ is measured from the upstream stagnation point and is positive in the clockwise direction. These results are compared with experimentally measured data $^{3}$ for the cylinder in Fig. 3(a). Recall that the flow was tripped using a transition strip between $\theta=50-60^{\circ}$ in the 
experimental case to force early transition on the cylinder surface. It is not practical in the current CFD codes to simulate transition in this manner, hence computations were run in a fully turbulent mode to simulate turbulent flow separation.

Several observations can be made by examining the results in Fig. 3(a). First we note that for the same turbulence model, either SST-QL or MDDES, both the FUN3D and CFL3D results agree well with each other. In general, all of the predicted results are in good agreement with the measured data on the front side of the cylinder as well as at the aft end. However, for the SST-QL and HRLES models, the suction peak is low compared to the measured value, indicating early flow separation. The suction peak is captured much better with the MDDES model, resulting in the best agreement with the measured pressure data. From these results, it is apparent that the separation point moves further aft on the cylinder with the MDDES turbulence model compared to the SST-QL and HRLES models.

The root mean square (rms) of the surface pressure perturbations are examined in Fig. 3(b), where the FUN3D solutions are compared with the experimental data and CFL3D results. It is noted that the FUN3D and CFL3D results with the same (SST-QL or MDDES) turbulence model are in excellent agreement with each other, as was the case for surface pressure. Overall agreement of the computed results with the measured data is quite good. In general, the results from the HRLES turbulence model compare at least as well or better with the experimental data as the results from the SST-QL model, which is derived from heuristic arguments and is strongly dependent on grid topology.

Next we examine the flow field in the wake region of the cylinder. Detailed PIV data for this case is available at a constant spanwise plane from the experimental work of Jenkins et al. ${ }^{3}$ Equivalent data from CFD analysis is obtained by averaging the time-averaged data along the spanwise direction. The time-averaged streamwise velocity contours based on FUN3D results with the SST-QL, HRLES and MDDES turbulence models shown in Fig. 4 are in overall good qualitative agreement with the experimental data. The main influence of the turbulence model appears to be on the size and extent of the reverse flow region. The SST-QL and HRLES models predict a larger recirculating region, whereas the MDDES model appears to be closer to experimental data in this respect. This is verified further by looking at the streamline plots shown in Fig. 5. The computational flow patterns seen in this figure are qualitatively similar to what has been observed in the experimental work of Jenkins et al. ${ }^{10}$ However, the reverse flow region based on SST-QL and HRLES results extend farther in the downstream direction compared to the measured data. This is not surprising, given that the flow separation occurs further upstream on the cylinder compared to the experimental value, and hence resulting in a wider and longer wake behind the cylinder. By contrast, the size and extent of the reverse flow region predicted by the MDDES model in the wake region is in better agreement with the measured data, as seen in Fig. 5.

Contour plots for the spanwise vorticity, "Z-vort", and the 2-D component of the turbulence kinetic energy representing $\left[1 / 2\left(u^{\prime 2}+v^{\prime 2}\right)\right]$, and referred as "2-D TKE" are shown in Figs. 6 and 7, respectively. In general, the computed results are in good agreement with the experimental data, with the MDDES results being closer in terms of the size and extent of the high intensity TKE region. The vorticity plots based on the FUN3D computations display well resolved shear layers off the cylinder surface and closely resemble the experimental results.

The time-averaged streamwise velocity and 2-D TKE results along the $y=0$ line downstream of the cylinder are shown in Fig. 8. Computational results for the streamwise velocity again indicate a larger reverse flow region compared with the measured data, with the MDDES results being in closer agreement with the measurements. The computed peak values of TKE are slightly higher than the measured data. Nonetheless these comparisons are considered encouraging. Based on the results shown in Fig. 8, it is clear that the FUN3D and CFL3D solutions for the same turbulence model (SST-QL or MDDES) are in excellent agreement with each other.

The surface pressure power spectral density (PSD) data extracted from the CFD simulations are compared with the experimental data at an azimuthal angle of $135^{\circ}$ on the cylinder in Fig. 9. The agreement between the computed results with measurements is quite reasonable for lower frequencies. The flatter response seen in the experimental data for frequencies above $400 \mathrm{~Hz}$ may be caused by the transition strip in the experiments. The predicted values of the primary tone frequencies are slightly lower than the experimental values for the SST-QL and HRLES models, whereas for the MDDES model the reverse is true. The strength of the tones is also underpredicted, but this is partially an artifact of the relatively large bin width $(15 \mathrm{~Hz})$ in the CFD results compared with $3.25 \mathrm{~Hz}$ in the experiment. It is noted that the larger bin width for CFD solutions was used because of the limitations imposed by the available data in these computations.

\section{VI.B. Tandem Cylinders}

The next test case chosen for this study corresponds to the tandem cylinder configuration of Refs. $(3-6,10)$ where the two cylinders are separated by a distance of 3.7 cylinder diameters. Lockard et al. ${ }^{6}$ have presented results for this configuration on multi-block structured grids of varying grid densities. The medium density grid with a span of 3 cylinder diameters (3D) and 97 equally spaced spanwise planes was shown to capture most significant aspects of pertinent flow physics in Ref. 6, and, hence, this grid was chosen as a baseline grid for the CFL3D code in the current study. As mentioned earlier, this structured grid could not be used directly in the FUN3D unstructured grid code because of the 
hanging nodes in the patched (non point-matched) regions of the grid. Instead the AFLR2 grid generation code ${ }^{17}$ was used to create the unstructured grids used here. A sectional plot of the unstructured grid is shown in Fig. 1(b). The test conditions used for the computations are identical to the single cylinder case. The results obtained with the SST-QL and MDDES turbulence models in the CFL3D code, and with the SST-QL and HRLES models in the FUN3D code are discussed in the following paragraphs. We were not able to obtain statistically meaningful solutions with the MDDES turbulence model in FUN3D code on this grid, and effort is undergoing to isolate the cause of this discrepancy.

The time-averaged surface pressure distributions are presented in Fig. 10 along with the experimental data. The computed pressures are in good agreement with each other and the experimental data for the front cylinder. The MDDES results with CFL3D capture both the suction peak and base recovery on the front cylinder observed in the experiments. All of the other models produce results similar to each other. For the rear cylinder, the agreement between the CFL3D and FUN3D codes using the SST-QL model is deemed fair, considering the differences in the grids. Regardless of the turbulence model, the predicted suction peaks are much smaller than the corresponding measurements. In Ref. 6 , Lockard et al. have shown that the agreement with the experimental data improved marginally for the SST-QL results if a larger span $($ span $>3 \mathrm{D})$ was used in the numerical simulations.

The rms of the surface pressure perturbations are examined in Fig. 11, where the CFD solutions are compared with the experimental data. We have included CFL3D and FUN3D results with the SST-QL model, along with the results from the HRLES model for FUN3D and the MDDES model for CFL3D. The difference between the SST-QL and HRLES results are relatively small. Again the agreement between the two CFD codes for the SST-QL model is considered quite good, considering the fact that very different grids were used for these computations. For the front cylinder, the predicted pressure perturbations with the SST-QL and HRLES models are higher than the measurements, whereas the MDDES model predicts lower values for the perturbation pressure. For the rear cylinder, the computational results are in fair agreement with the data except near the top and bottom portions of the cylinder, where the location and influence of the flow separation are missed.

The contour plots for the 2-D TKE from the present computations are compared with the experimental data in the gap region between the cylinders and in the wake region aft of rear cylinder in Figs. 12 and 13, respectively. Although the size and extent of the high intensity TKE region in FUN3D results are somewhat large compared to the measurements, the computed results display most of the salient features observed in experimental data. The computational results along the wake centerline are compared with the experimental data in Fig. 14. The CFD solutions follow the overall trends observed experimentally, and the location of predicted peaks for TKE are in good agreement in the gap region. The SST-QL and HRLES predict higher levels of TKE, whereas the reverse is true for the MDDES model. In the wake region of the rear cylinder, all of the CFD solutions underpredict the peak levels of TKE, and these solutions show less sensitivity to turbulence modeling.

The power spectral density (PSD) data extracted from the FUN3D and CFL3D simulations are compared with the experimental data at azimuthal angle of $135^{\circ}$ on the front cylinder and $45^{\circ}$ on the rear cylinder in Fig. 15. Except for slight offsets, the CFD results compare quite well with the experimental data. The results from the two CFD codes are generally in good agreement with each other. The primary effect of turbulence model is a shift in the tonal frequency. For the SST-QL model, FUN3D and CFL3D results compare well with each other.

\section{Concluding Remarks}

The unstructured grid flow solver, FUN3D is used to solve flow past circular cylinder configurations at a Reynolds number of $1.66 \times 10^{5}$ based on cylinder diameter. The resulting solutions are compared with experimental data from NASA Langley's BART and QFF wind tunnels, and solutions obtained with the structured grid code, CFL3D. Due to the aperiodic time-dependent nature of the flow, computations are performed over a large number of time steps to ensure statistical convergence of the flow field. An attempt is made to assess the sensitivity of the resulting solutions to changes in the turbulence model. Computations are also performed with two-equation based models (SST-QL and HRLES) and the one-equation based DDES model popular for such flow problems. Minor modifications are made to the DDES model in order to recover standard RANS solutions in the boundary layer region.

For the single cylinder configuration, excellent agreement is shown between the FUN3D and CFL3D solutions using the same turbulence models on identical grids, thus establishing the accuracy and applicability of the FUN3D solver for such problems. Detailed comparisons with the experimental data have been presented, and in general the computational results compare quite well with experimental data not only for surface pressure and perturbation pressures, but also for various flow field quantities, such as the turbulence kinetic energy and spanwise vorticity. The results obtained with the MDDES model are in closer agreement with the experimental data for this case. 
For the tandem cylinders, FUN3D solutions are obtained on an unstructured grid comparable in resolution in the near field to the structured grid used for CFL3D computations. The agreement between the solutions obtained from these two codes for the same turbulence model is good in general. The agreement with the experimental data for time-averaged and perturbation quantities is considered fair. For the power spectral density, the FUN3D and CFL3D solutions are in good agreement with each other and correlate well with the measured data. No clear trend emerges regarding the choice of a suitable turbulence model for this case.

Further work should continue to quantify the effect of grid resolution and grid type, especially for the tandem cylinders. Work should also continue on examining the effect of the MDDES model parameter $C_{M D D}$ on resulting solutions.

\section{Acknowledgments}

This work was supported by NASA's Fundamental Aerodynamics Program through the Subsonic Fixed-Wing Project. The authors are deeply indebted to Dr. Eric J. Nielsen of NASA Langley Research Center for providing invaluable guidance and support related to the mixed element capability and periodic boundary conditions for the FUN3D code.

\section{References}

\footnotetext{
${ }^{1}$ FUN3D Web page: http://fun3d.larc.nasa.gov, March 2010.

${ }^{2}$ Rumsey, C., Biedron, R., and Thomas, J., “CFL3D: Its History and Some Recent Applications,” NASA TM 112861, May 1997, presented at the Godonov's Method for Gas Dynamics Symposium, Ann Arbor, MI.

${ }^{3}$ Jenkins, L. N., Neuhart, D. H., McGinley, C. B., Choudhari, M. M., and Khorrami, M. R., "Measurements of Unsteady Wake Interference Between Tandem Cylinders," AIAA Paper 2006-3202, June 2006.

${ }^{4}$ Hutcheson, F. V. and Brooks, T., "Noise Radiation from Single and Multiple Rod Configurations," AIAA Paper 2006-2629, May 2006.

${ }^{5}$ Khorrami, M. R., Choudhari, M. M., Jenkins, L. N., and McGinley, C. B., "Unsteady Flowfield Around Tandem Cylinders as Prototype for Component Interaction in Airframe Noise," AIAA Paper 2005-2866, 11 th AIAA/CEAS Aeroacoustics Conference, Monterey, CA., May 23-25, 2005.

${ }^{6}$ Lockard, D. P., Khorrami, M. R., Choudhari, M., Hutcheson, F., Brooks, T., and Stead, D., “Tandem Cylinder Noise Predictions,” AIAA Paper 2007-3450, May 2007.

${ }^{7}$ Spalart, P. and Allmaras, S., "A one-equation turbulence model for aerodynamic flows," Recherche Aerospatiale, , No. 1, 1994, pp. 5-21. 1605.

${ }^{8}$ Menter, F. R., "Two-equation Eddy-viscosity Turbulence Models for Engineering Applications," AIAA Journal, Vol. 32, No. 8, 1994, pp. 1598-

${ }^{9}$ Khorrami, M. R., Lockard, D. P., Choudhari, M. M., Jenkins, L. N., Neuhart, D. H., and McGinley, C. B., "Simulation of Bluff Body Flow Interaction for Noise Source Modeling," AIAA Paper 2006-3203, June 2006.

${ }^{10}$ Jenkins, L. N., Khorrami, M. R., Choudhari, M. M., and McGinley, C. B., "Characteristization of Unsteady Flow Structures Around Tandem Cylinders for Component Interaction Studies in Airframe Noise," AIAA Paper 2005-2812, May 2005.

${ }^{11}$ Roe, P. L., "Approximate Riemann solvers, parameter vectors, and difference schemes," Journal of Computational Physics, Vol. 43, 1981, pp. 357-372.

${ }^{12}$ Anderson, W. K. and Bonhaus, D. L., "An Implicit Upwind Algorithm for Computing Turbulent Flows on Unstructured Grids," Computers and Fluids, Vol. 23, No. 1, 1994, pp. 1-21.

${ }^{13}$ Nielsen, E., Lu, J., Park, M., and Darmofal, D., "An Implicit, Exact Dual Adjoint Solution Method for Turbulent Flows on Unstructured Grids," Computers and Fluids, Vol. 33, No. 9, 2003, pp. 1131-1155.

${ }^{14}$ Spalart, P. R., "Young Person's Guide to Detached-Eddy Simulation Grids," NASA CR-211032, 2001.

${ }^{15}$ Travin, A., Shur, M. L., Strelets, M., and Spalart, P. R., "Detached-Eddy Simulations Past a Circular Cylinder," Flow, Turbulence and Combustion, Vol. 63, 1999, pp. 293-313.

${ }^{16}$ Vatsa, V. N. and Singer, B., "Evaluation of a Second-Order Accurate Navier-Stokes Code for Detached Eddy Simulation Past a Circular Cylinder," AIAA Paper 2003-4085, June 2003.

${ }^{17}$ Marcum, D., "Generation of Unstructured Grids for Viscous Flow Applications," AIAA Paper 1995-0212, January 1995.

${ }^{18}$ Lynch, C. and Smith, M. J., "Hybrid RANS-LES Turbulence Models on Unstructured Grids," AIAA Paper 2008-3854, June 2008.

${ }^{19}$ Spalart, P. R., Deck, S., Shur, M. L., Squires, K. D., Strelets, M. K., and Travin, A., "A new version of detached-eddy simulation, resistant to ambiguous grid densities," Theoretical Computational Fluid Dynamics, Vol. 20, 2006, pp. 181-195.

${ }^{20}$ Vatsa, V., Carpenter, M., and Lockard, D., "Re-evaluation of an Optimized Second Order Backward Difference (BDF2OPT) Scheme for Unsteady Flow Applications," AIAA Paper 2010-0122, January 2010.
} 


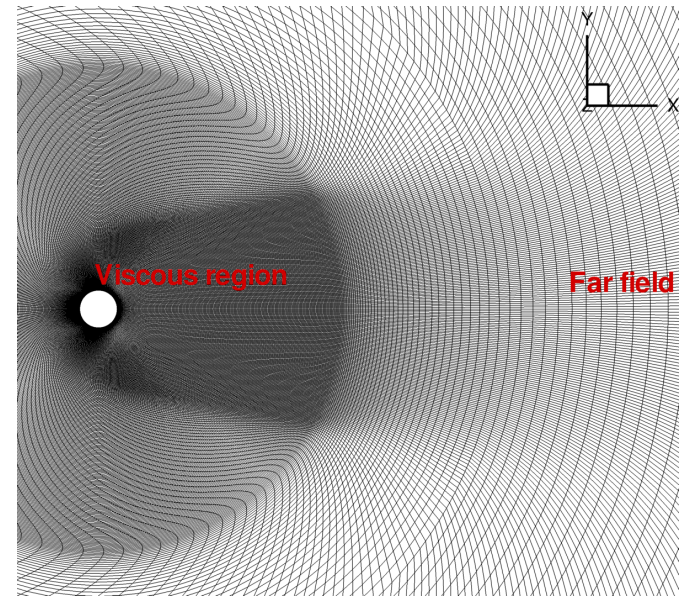

(a) Single cylinder

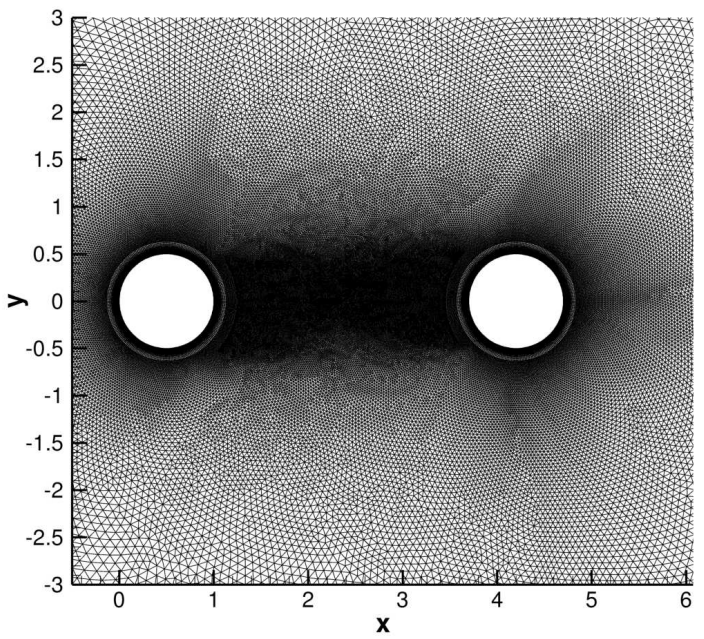

(b) Tandem cylinders

Figure 1. Partial view of computational grid

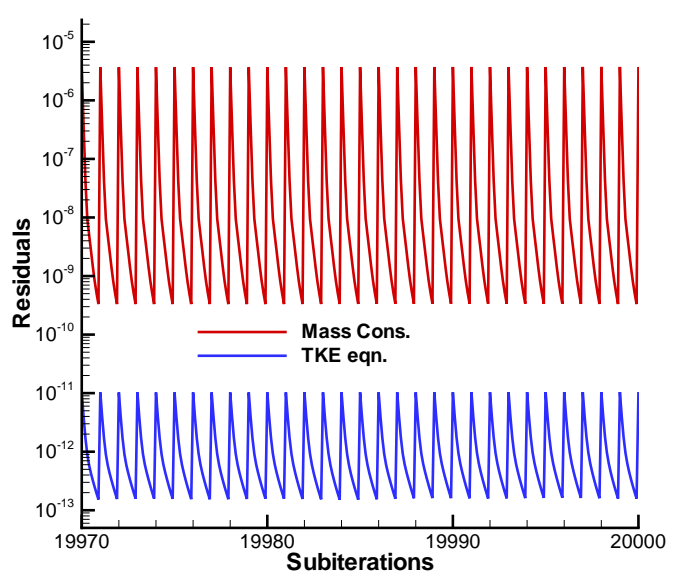

(a) Subiteration convergence

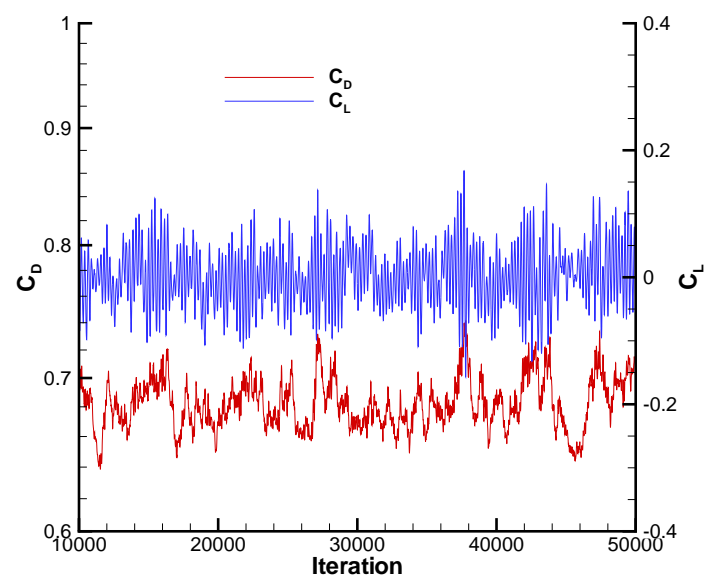

(b) Lift and drag history

Figure 2. Convergence history for single cylinder 


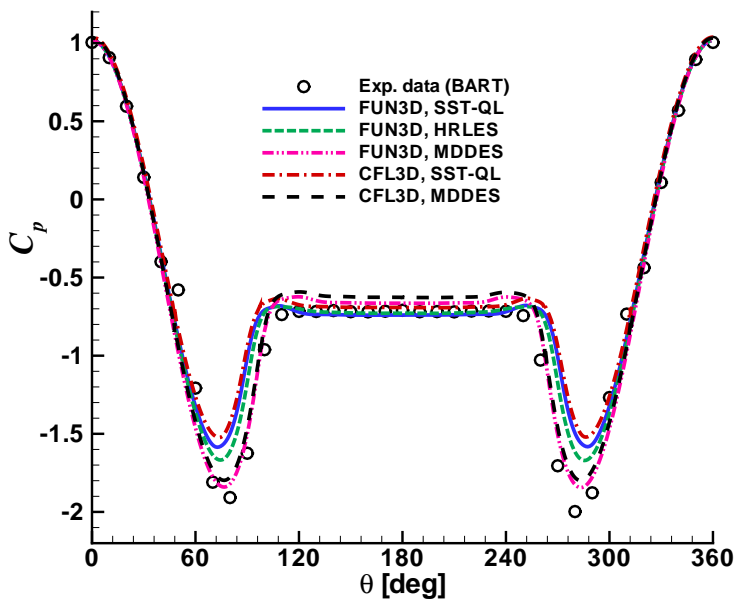

(a) time-averaged surface pressure coefficient

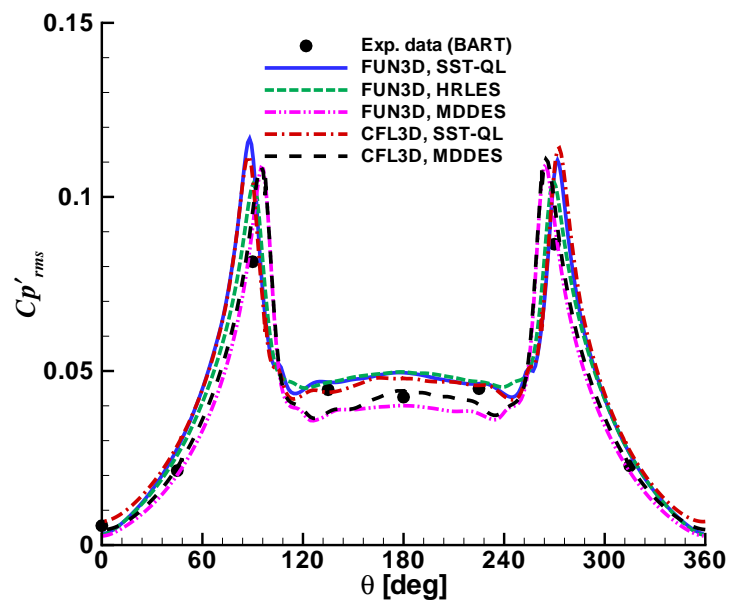

(b) perturbation pressure coefficient

Figure 3. Comparison of surface pressures for single cylinder

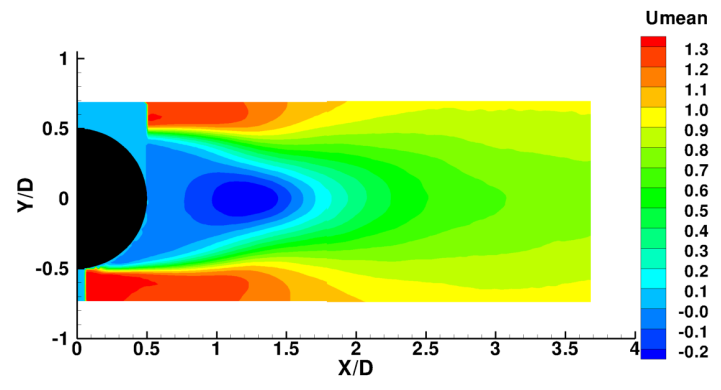

(a) Experimental data

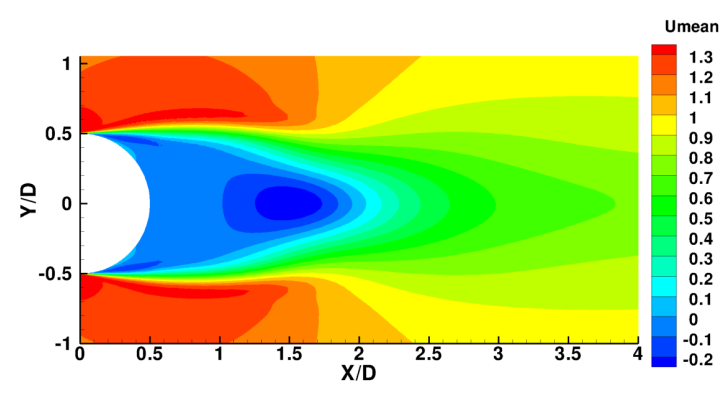

(c) HRLES Model

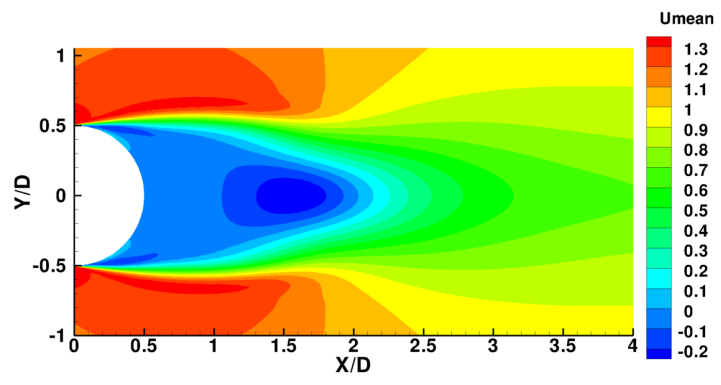

(b) SST-QL Model

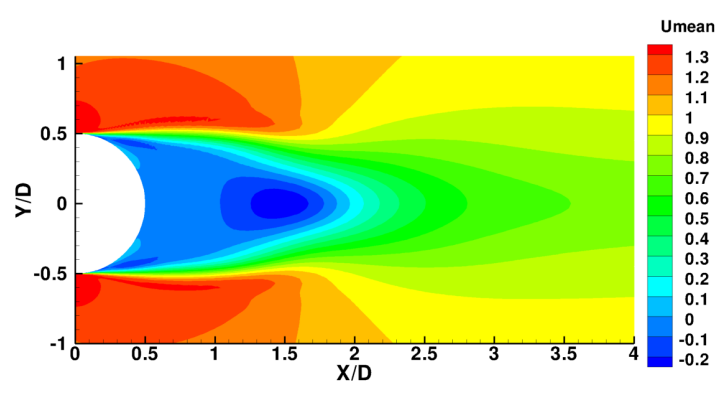

(d) MDDES Model

Figure 4. Time-averaged streamwise velocity contours for single cylinder 


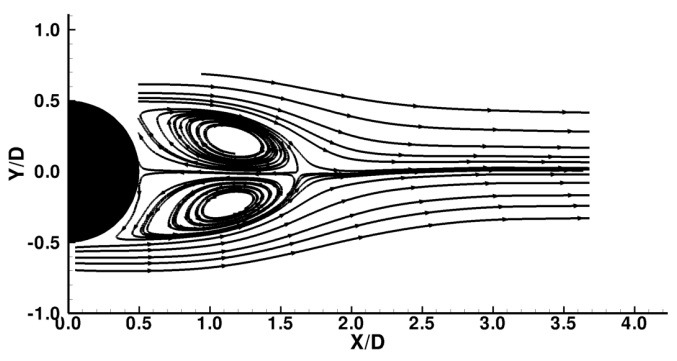

(a) Experimental data

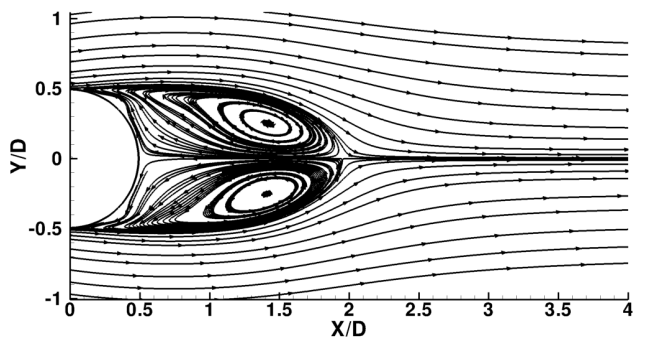

(c) HRLES Model

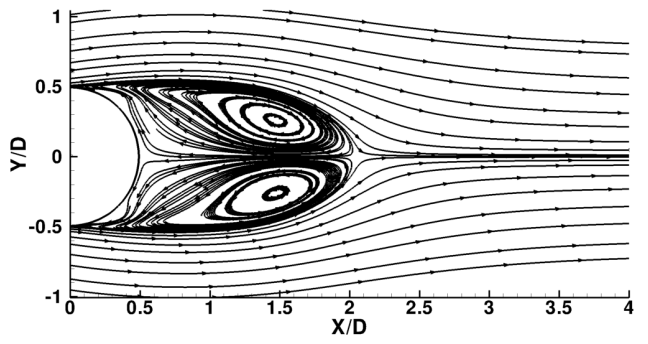

(b) SST-QL Model

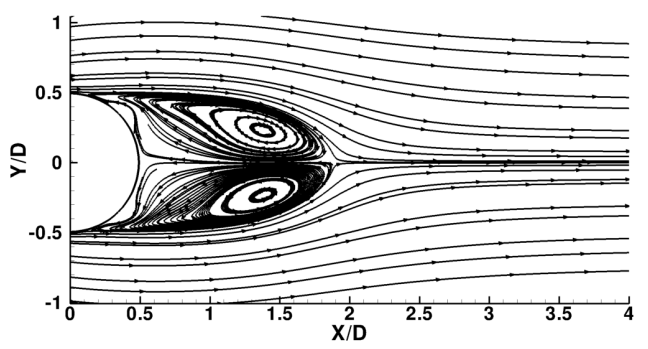

(d) MDDES Model

Figure 5. Mean flow streamlines for single cylinder

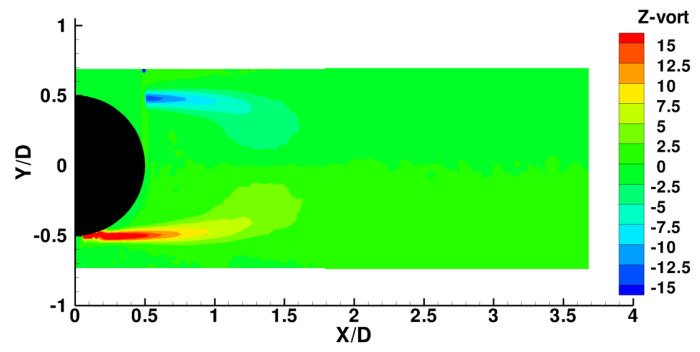

(a) Experimental data

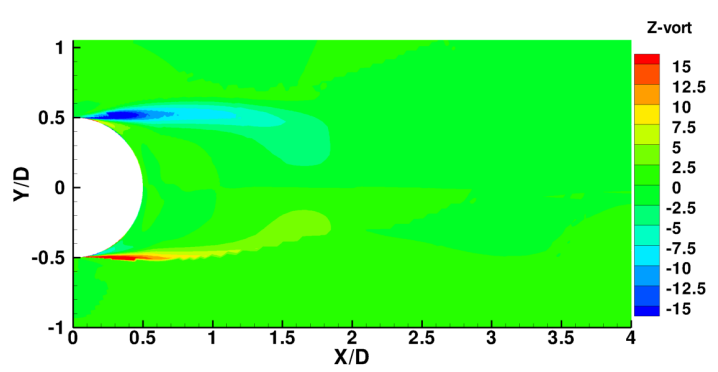

(c) HRLES Model

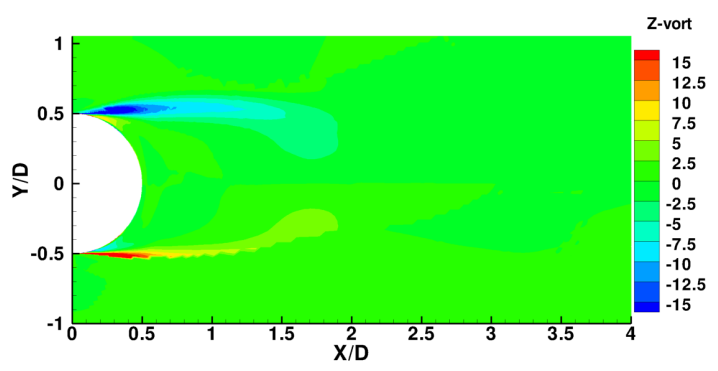

(b) SST-QL Model

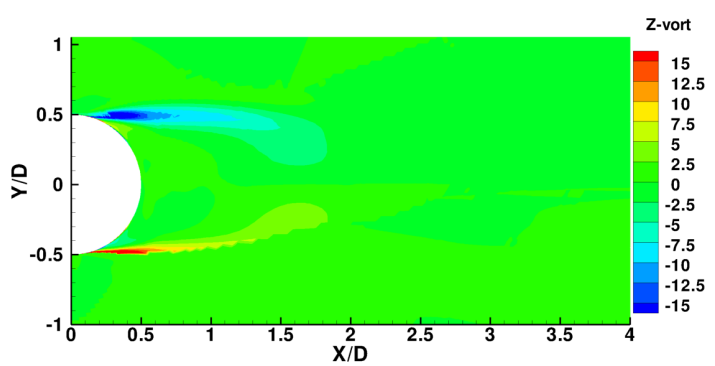

(d) MDDES Model

Figure 6. Spanwise vorticity contours for single cylinder 


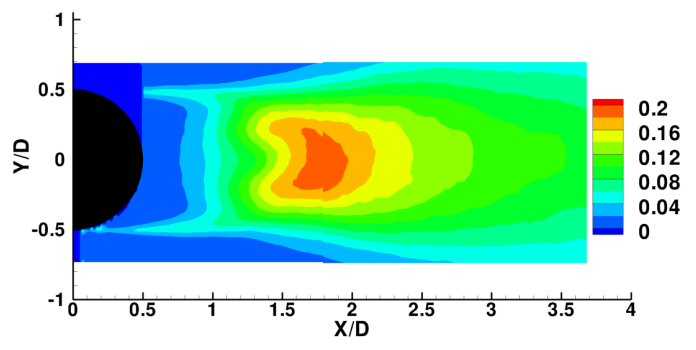

(a) Experimental data

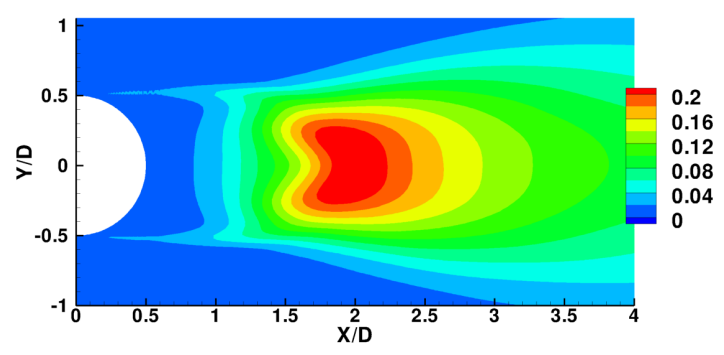

(c) HRLES Model

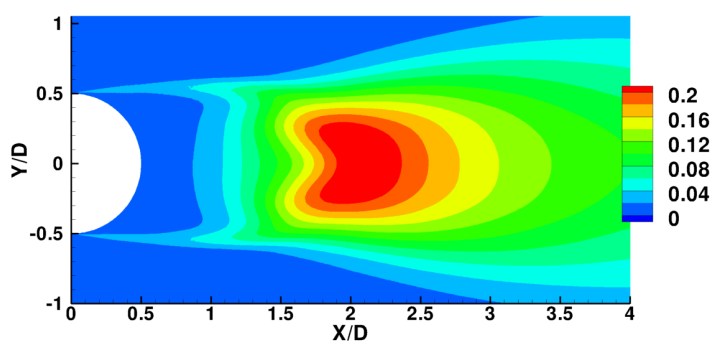

(b) SST-QL Model

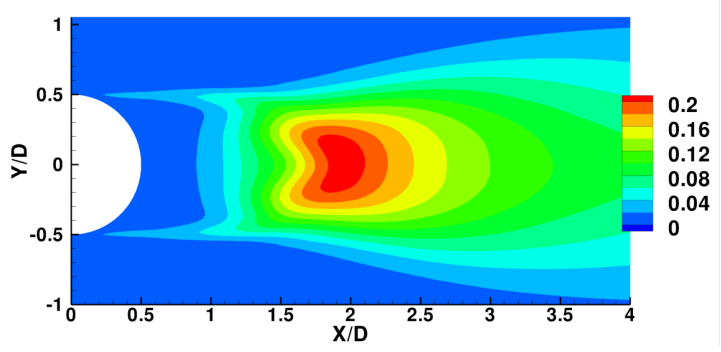

(d) MDDES Model

Figure 7. 2-D TKE contours for single cylinder

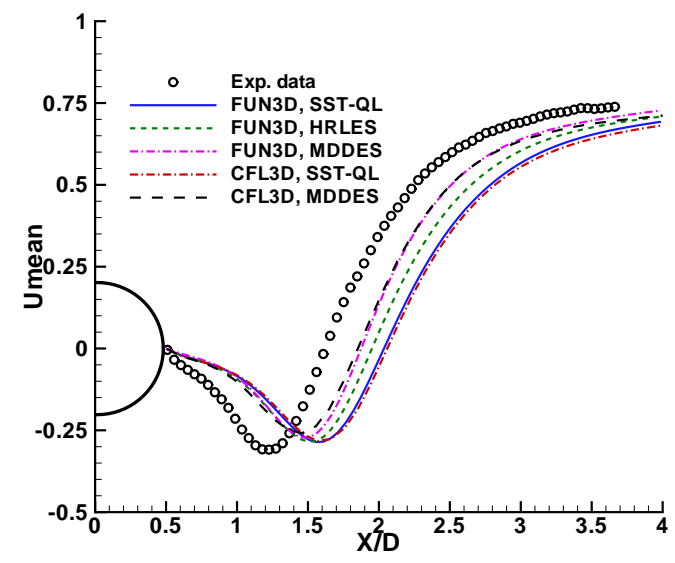

(a) streamwise mean velocity

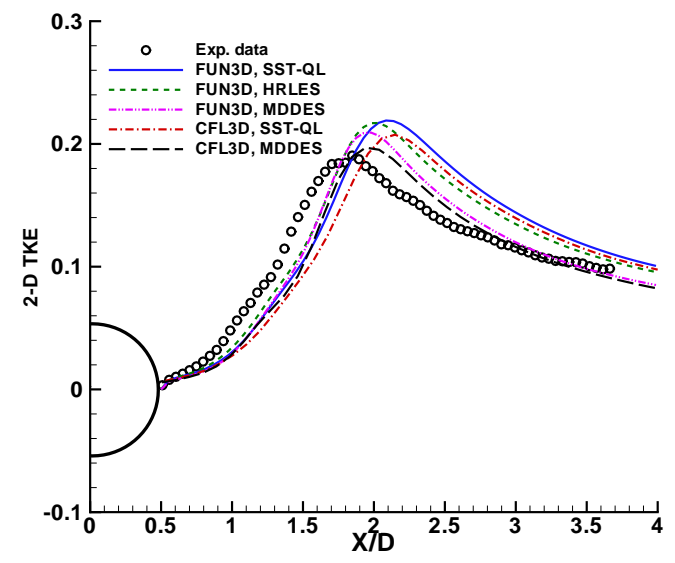

(b) 2-D TKE

Figure 8. Data comparison along wake centerline, single cylinder 


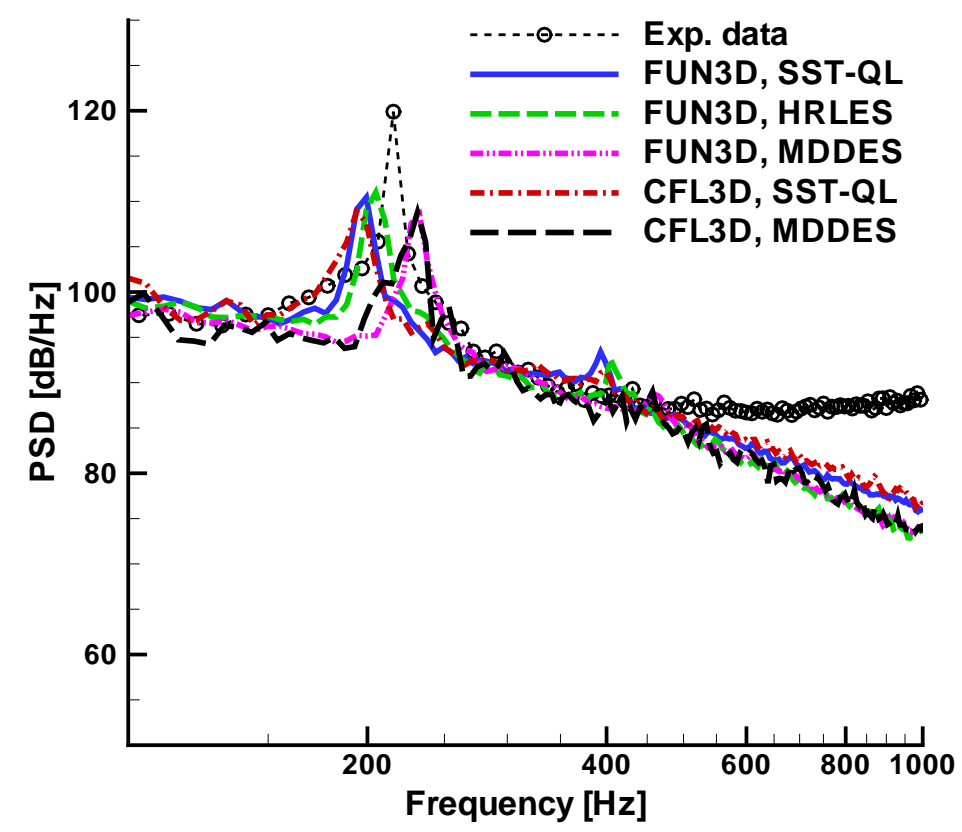

Figure 9. Power spectral density comparisons for single cylinder, $\theta=135^{0}$

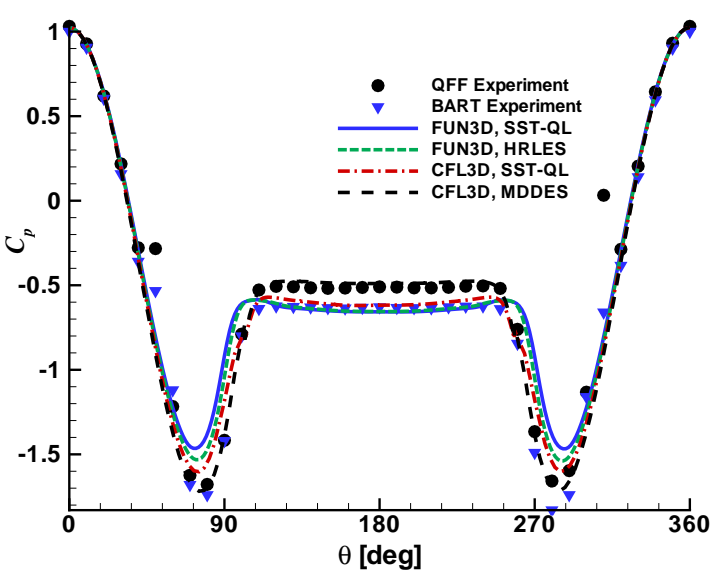

(a) Front cylinder

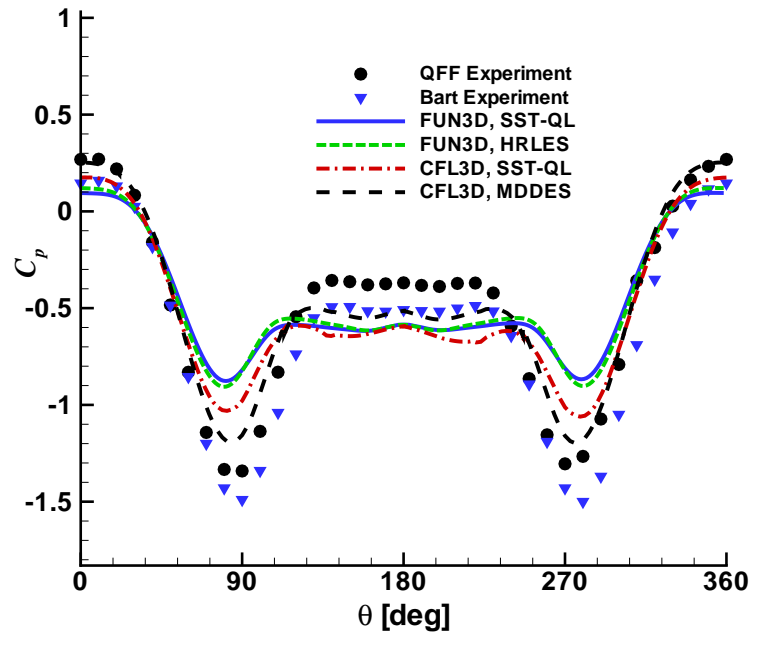

(b) Rear cylinder

Figure 10. Comparison of time-averaged surface pressures for tandem cylinders 


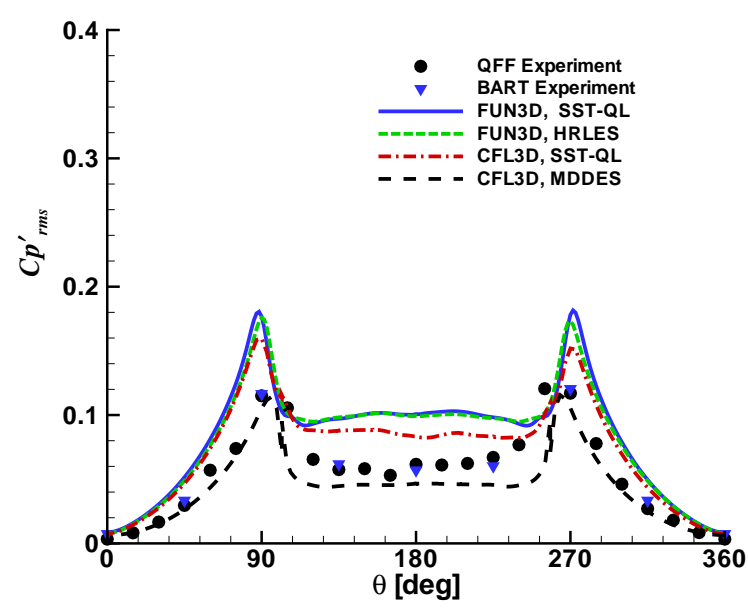

(a) Front cylinder

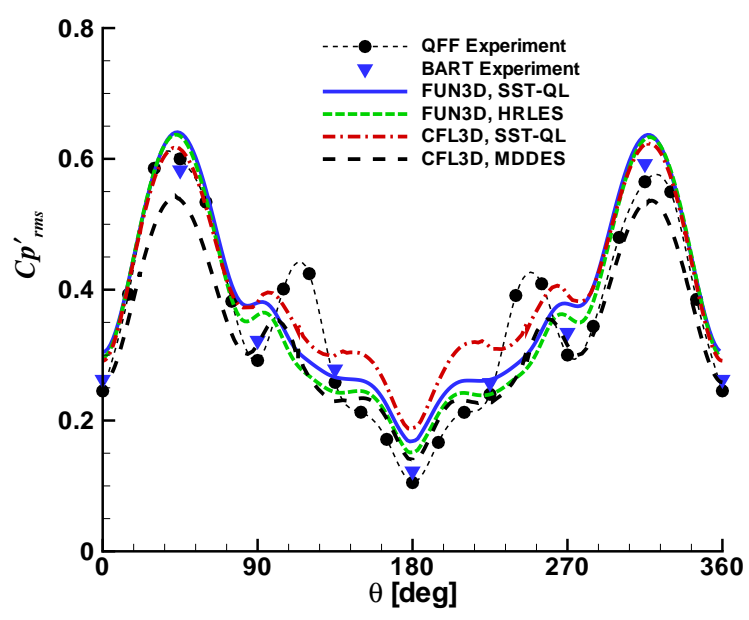

(b) Rear cylinder

Figure 11. Comparison of perturbation surface pressures for tandem cylinders

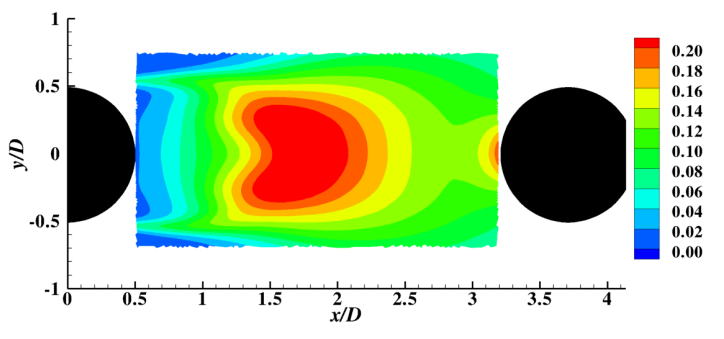

(a) FUN3D, SST-QL Model

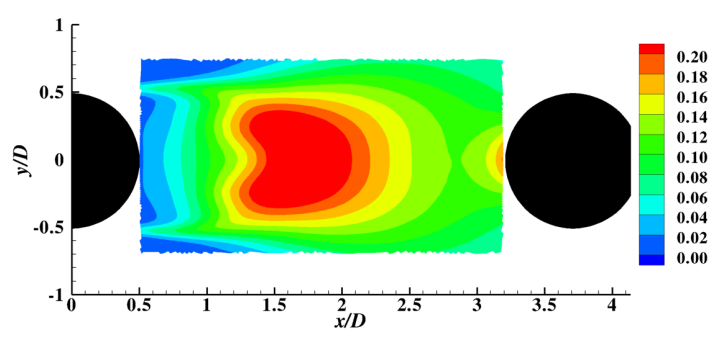

(b) FUN3D, HRLES Model

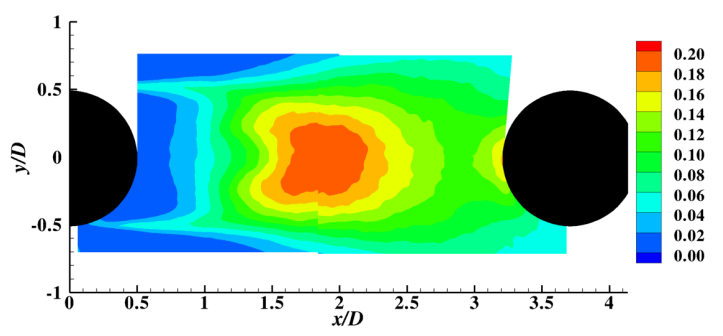

(c) Experimental data

Figure 12. 2-D TKE contours in gap region of tandem cylinders 


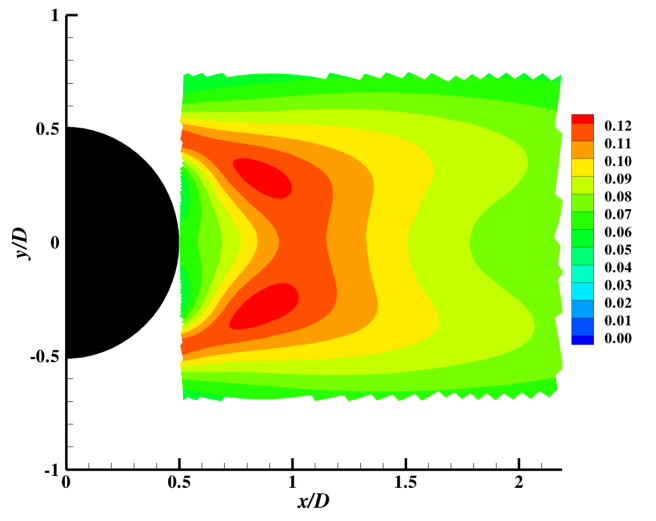

(a) FUN3D, SST-QL Model

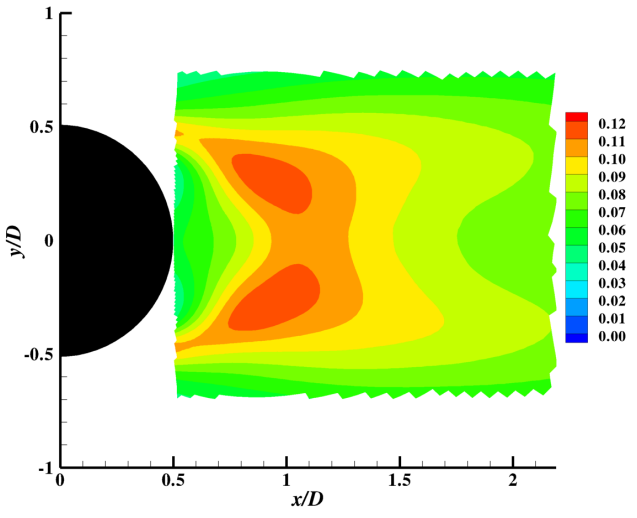

(b) FUN3D, HRLES Model

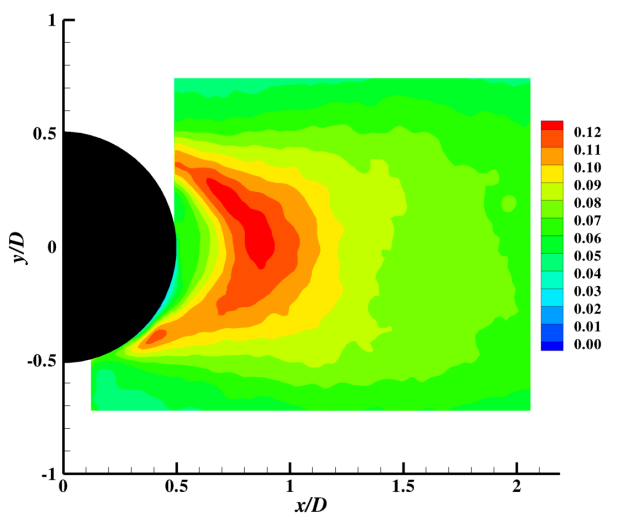

(c) Experimental data

Figure 13. 2-D TKE contours in wake region of rear cylinder

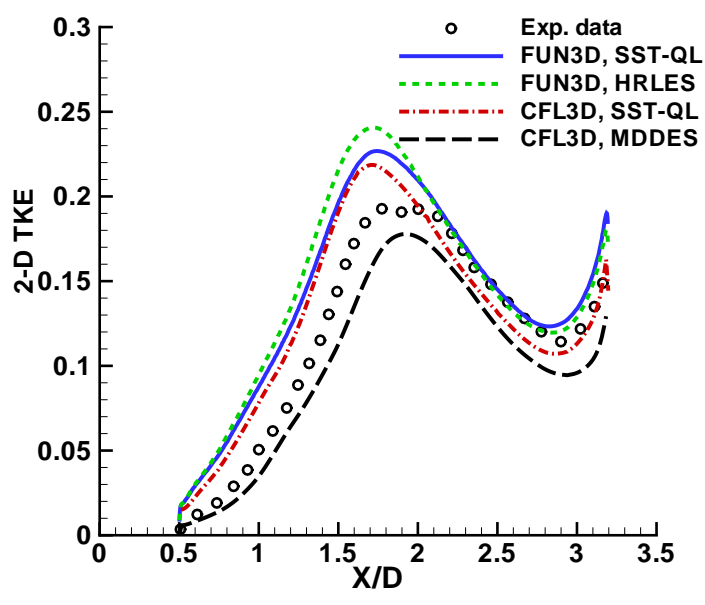

(a) gap region

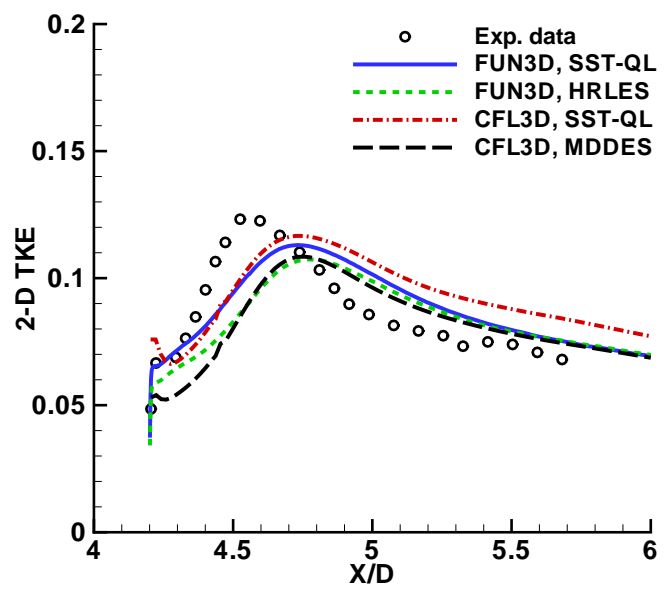

(b) aft of rear cylinder

Figure 14. 2-D TKE comparisons along wake centerline, tandem cylinder 


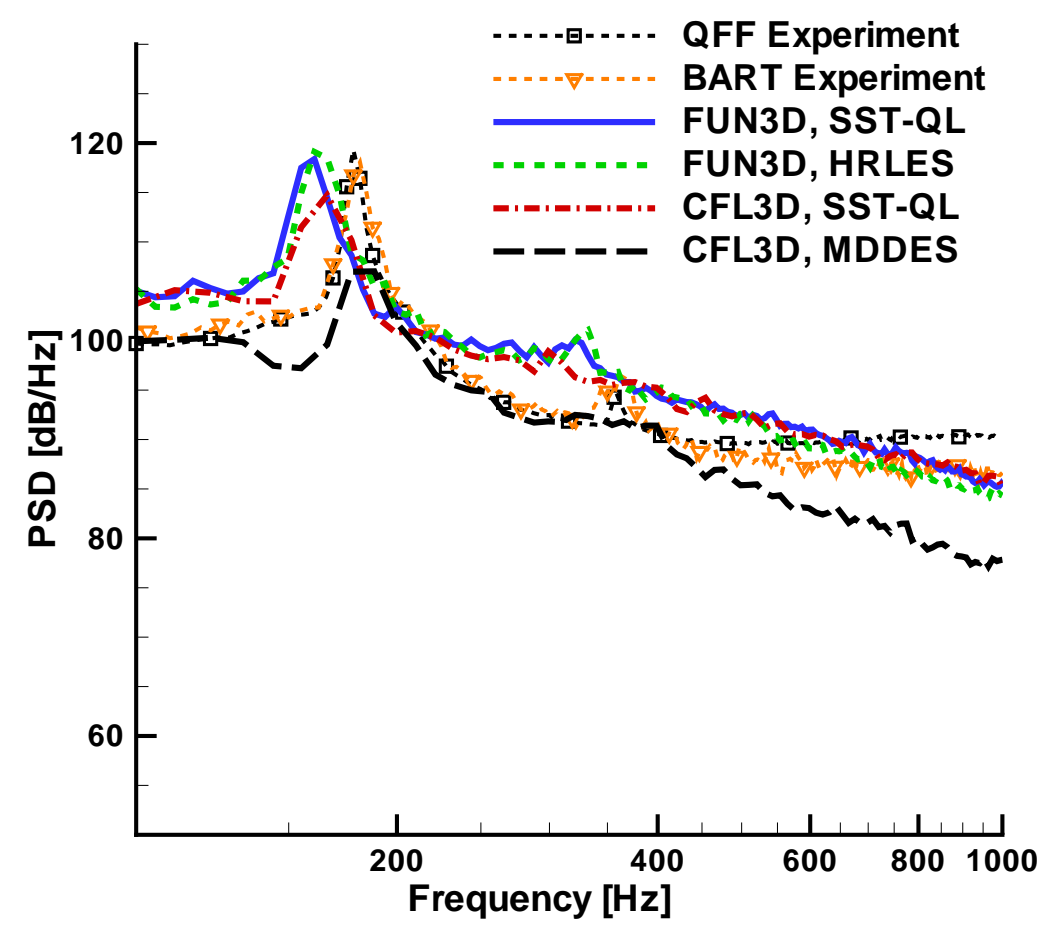

(a) $\theta=135^{\circ}$, upstream cylinder

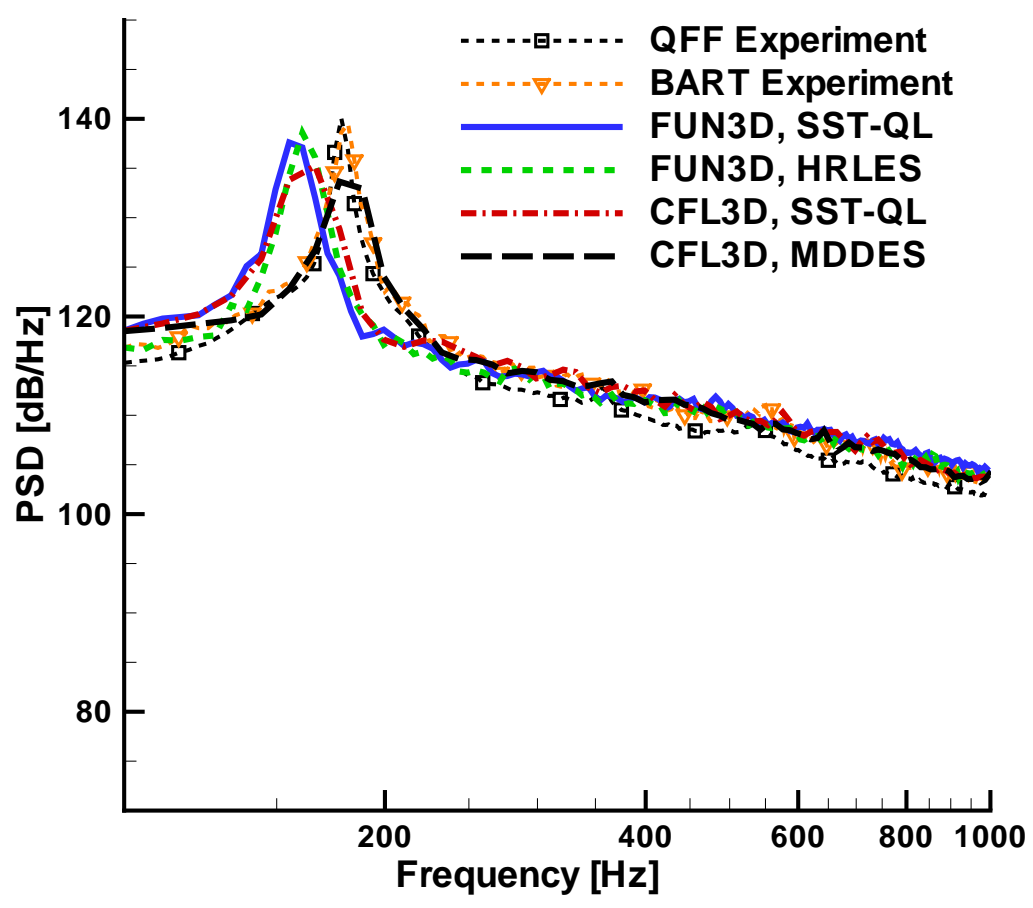

(b) $\theta=45^{\circ}$, rear cylinder

Figure 15. Power spectral density comparisons for tandem cylinders 